\title{
ADAPTIVE GEOMETRIC INTEGRATORS FOR HAMILTONIAN PROBLEMS WITH APPROXIMATE SCALE INVARIANCE*
}

\author{
S. BLANES ${ }^{\dagger}$ AND C. J. BUDD $\ddagger$
}

\begin{abstract}
We consider adaptive geometric integrators for the numerical integration of Hamiltonian systems with greatly varying time scales. A time regularization is considered using either the Sundman or the Poincaré transformation. In the latter case, this gives a new Hamiltonian which is usually separable, but with one of the parts not always exactly solvable. This system can be numerically integrated with a splitting scheme where each part can be computed using a symplectic implicit or explicit method, preserving the qualitative properties of the exact solution. In this case, a backward error analysis for the numerical integration is presented. For a one-dimensional near singular problem, this analysis reveals a strong dependence of the performance of the method with the choice of the monitor function $g$, which is also observed when using other symmetric nonsymplectic integrators. We also show how this dependence greatly increases with the order of the numerical integrator used. The optimal choice corresponds to the function $g$, which nearly preserves the scaling invariance of the system. Numerical examples supporting this result are presented. In some cases a canonical transformation can also be considered, making the system more regular or easy to compute, and this is also illustrated with some examples.
\end{abstract}

Key words. Hamiltonian systems, symplectic integrators, variable time step, scaling invariance, canonical transformations

AMS subject classifications. 34A26, 65L05, 65P10, 70F16, 70H15

DOI. $10.1137 / \mathrm{S} 1064827502416630$

1. Introduction. When solving Hamiltonian systems of ordinary differential equations, certain qualitative properties of the evolution are important, and symplectic integrators have largely shown during the last decade to be superior to standard integrators $[36,10,20]$ when used with constant time step. In contrast, adaptive variable time step methods are often superior to fixed time step methods when applied to problems with varying evolutionary time scales. They lead to more regular problems with reduced local errors and with the effects of rounding error minimized. The results presented in the paper [8] demonstrate that adaptive methods can be especially effective when the underlying problem has a scaling structure. However, adaptive and symplectic methods have tended to sit uncomfortably together, with adaptivity often corrupting the powerful long time error estimates obtained for fixed time step symplectic methods [36]. Attempts to rectify this problem, which we will describe, have tended to result in either complex and hard to use algorithms or low order methods. It is also not clear in many of these methods what choice should be made of the adaptive procedure, and how this choice affects the performance of a numerical integrator of a given order. We present in this paper a technique for identifying a natural adaptive procedure based upon identifying the evolutionary scalings of the system, and then implementing this, using a combination of a Sundman and a Poincaré transform, for

\footnotetext{
* Received by the editors October, 28, 2002; accepted for publication (in revised form) December, 31, 2003; published electronically March 11, 2005. The work of both authors has been partially supported by the TMR program through grant EC-12334303730.

http://www.siam.org/journals/sisc/26-4/41663.html

$\dagger$ Instituto de Matemàtica Multidisciplinar, Universidad Politècnica de Valencia, Spain (sblanes@ mat.uji.es). This author's work has been partially supported by the Ministerio de Ciencia y Tecnología through a contract from the program Ramón y Cajal (2001) and project BFM2001-0262.

${ }_{\ddagger}^{\ddagger}$ Department of Mathematics, University of Bath, Claverton Down, Bath BA2 7AY, UK (cjb@maths.bath.ac.uk).
} 
Hamiltonian problems. This procedure gives the smallest error in the Hamiltonian over a class of adaptive methods for both problems with an exact scaling law and for others where the scaling law applies approximately at points where the solution is changing most rapidly. If the transformed problem is separable with each part exactly solvable (or accurately and efficiently approximated using explicit or implicit symplectic integrators) then applying splitting methods leads to adaptive, symplectic methods of arbitrary order, which are also invariant under changes of scale. In addition, backward error analysis can be applied to analyze these methods and gives a valuable insight about the importance of the choice of the monitor function for the performance of a method.

Finally, in some cases it is possible to consider a canonical transformation (CT) such that the rescaled system turns into a system which is more regular and easy or cheap to compute, and this allows an improvement of the performance of the integrator. This is always possible for the one-dimensional problem, but only in some cases at higher dimensions, an important case being the Levi-Civita (LC) and a generalization to it $[2,24,38]$.

1.1. Symplectic methods. Given the Hamiltonian $H(\mathbf{q}, \mathbf{p})$, an $n$th order symplectic integrator (SI) used with a fixed time step $h$ solves a discrete system which corresponds exactly (up to exponentially small terms in $h$ ) to the solutions of a perturbed Hamiltonian system with constant Hamiltonian $H(\mathbf{q}, \mathbf{p})+\delta H(\mathbf{q}, \mathbf{p}, h)$, where $\delta H=\mathcal{O}\left(h^{n}\right)$. If $\delta H$ is small enough, we can expect that both systems will share the same qualitative properties. This observation explains, in part, the success of symplectic methods and their wide use in simulating Hamiltonian systems. Since the appearance of the papers by Creutz and Gocksch [16], Yoshida [44], and Suzuki [40], considerable effort has been put into obtaining more efficient, explicit, constant time step symplectic integrators [30]. Particularly interesting are: symplectic partitioned Runge-Kutta (PRK) methods $[28,3,6]$ for separable systems like $H=T(\mathbf{p})+V(\mathbf{q})$; symplectic Runge-Kutta-Nyström (RKN) methods [14, 28, 5, 6] for examples where $T$ is quadratic in momenta; and near-integrable systems $H=H_{0}+\epsilon H_{1}$, where both $H_{0}$ and $H_{1}$ are exactly solvable or easy to approximate, and $\epsilon$ is a small parameter $[43,29,4]$. Crucial to these methods is the separability of the Hamiltonian which allows the use of explicit methods. These integrators have proved to be highly competitive for each family of problems, being cheap to use (as they are explicit) and having excellent error bounds over long integration times.

1.2. Variable time step methods. If a dynamical system can evolve either rapidly or slowly along different regions of its trajectory, a standard technique for optimizing the performance of an integrator is, for traditional methods, to introduce a variable time stepping strategy into the algorithm. The choice of time step is often dictated by an estimate of the local truncation error of the method given, for example, by the Milne device or the Zadunaisky estimate [23]. As an example of where such methods are needed for a Hamiltonian problem, consider the many body problem, in which near collisions lead to rapid changes in the solution over small time scales. Adaptivity can markedly increase the integration accuracy of a numerical procedure over a short time scale, although it may not improve its accuracy over long time intervals. In an attempt to overcome the latter problem, it is desirable to combine the short term accuracy of an adaptive method with the long time accuracy of a symplectic method. However, this synthesis of techniques has proved difficult to achieve. When standard techniques for changing the time step were included in a symplectic integrator, it was found [36] (see also $[13,17,37]$ ) that several of the 
good properties of symplectic methods were lost. These integrators still preserve symplecticity, but now, roughly speaking, they are exactly solving a perturbed timedependent Hamiltonian, $H(\mathbf{q}, \mathbf{p})+\delta \tilde{H}(\mathbf{q}, \mathbf{p}, t)$. If the time step is not changed properly, secular terms will appear, $\delta \tilde{H}$ will grow with $t$, and the error in energy and positions will grow similarly to standard nonsymplectic integrators. More recently, this problem has been reconsidered, and integrators with variable time step and nonsecular terms have been obtained. The key behind these methods is to introduce a regularization of the time (often by using a Sundman transformation). This technique has been successfully used for many years in celestial mechanics for solving the Kepler problem [42]. The drawback is that separable systems usually turn into nonseparable (or not easily separable), and the highly efficient explicit methods previously mentioned cannot be used any longer. Several alternatives have been recently considered in the literature and some will be described in more detail in section 2 .

1.3. Scaling invariance. Many Hamiltonian problems, such as the Kepler problem, are invariant up to changes in the scale of both time and the solution. Whilst many other systems are not exactly scaling invariant, close to (hard to compute) almost singular events such as near collisions are often approximately scaling invariant. Exploiting scaling invariance through a suitably chosen adaptive time stepping strategy to lead the scale invariant numerical schemes with the same scaling invariance as the underlying equations has proved to be a very useful property for numerically solving both partial and ordinary differential equations $[1,11,8,9,33]$. For certain initial conditions, scaling invariant systems admit self-similar solutions (i.e., solutions which map to themselves under a change of scale) which can be attractors for many other solutions starting from more general boundary conditions. It has been shown [8] that multi-step and Runge-Kutta integrators for scale invariant ODEs admit discrete self-similar solutions obtained by group reductions of the discretization of the original problem. These discrete self-similar solutions have the very desirable properties of (i) uniformly approximating the true self-similar solution for all times, and (ii) retaining the stability of the underlying self-similar solution and, hence, describing correctly the asymptotic evolution of the whole ODE system. In a sense, such performance of an adaptive method is optimal for this particular class of solutions. The principal drawback of the methods implemented in [8] is that they involve enlarging the original system, they can be implicit, and they are not necessarily symplectic. Furthermore, there are many problems which are unchanged under scaling but which posses solutions which are not self-similar. A good example is the Kepler problem, which admits nonself-similar periodic solutions which can be transformed into other periodic solutions through the action of Kepler's third law. However, if the periodic orbit has high eccentricity with close approaches to the central body, then as it approaches, the natural time scale for its evolution is closely linked to its spatial position. Scaling can guide the correct choice of our adaptive method in this case.

When it is possible to combine both the scaling and the Hamiltonian structure of the original problem into a numerical integrator, we end up with an integrator which keeps the good behavior for long integration times, has the benefits of a variable time step algorithm, and can accept scaling invariant solution with a constant relative error. The construction and analysis of these is the objective of this paper.

1.4. An example. All of the above discussion, and indeed the remainder of this paper, can be both summarized and motivated through the following example. As remarked above, we hope to achieve, by combining adaptive and symplectic splitting methods with a choice of time step based on scaling invariance, a method which 


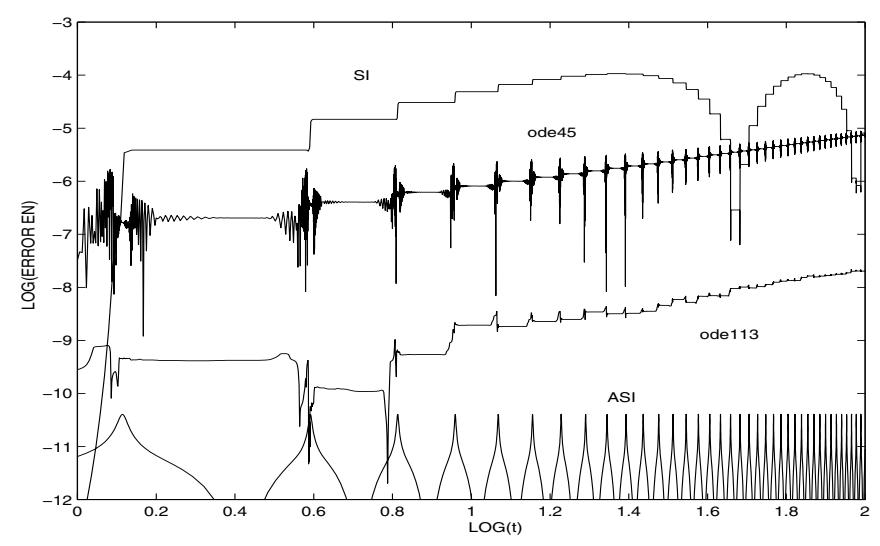

FIG. 1.1. Evolution of the error in energy for the Hamiltonian (1.1) in a $\log -\log$ scale using different integrators. The time step and tolerances are chosen such that all methods require approximately the same number of force evaluations. For a better illustration, SI has been computed with twice the number of evaluations.

is accurate on both short and long time scales. A problem in which all of these features are desirable is the Kepler problem, which possesses periodic orbits with close approaches. Such an orbit with a small angular momentum has a radial coordinate $q$ with associated momentum $p$ which evolves according to the Hamiltonian

$$
H=\frac{1}{2} p^{2}-\frac{1}{q}+\frac{\varepsilon}{q^{2}}
$$

where $\sqrt{\varepsilon}$ is the angular momentum. We now integrate this system, taking as initial conditions $\left(q_{0}, p_{0}\right)=(1,0)$, in the case of $\varepsilon=0.1$ over 40 periods using four different methods, namely, the Matlab integrators ode45 and ode113, an explicit sixth order RKN symplectic method with constant time step (SI), and the same method included as part of an adaptive integrator in which a scale invariant time transformation is employed (ASI). In each case the step size of each method was chosen so that approximately the same number of force evaluations was made during the integration. Since the value of $H$ is constant, we compute its numerical value at each step and measure its relative error with respect to the original value. Figure 1.1 shows the results obtained, which neatly illustrates all that we have said. The adaptive, nonsymplectic methods outperform the classical symplectic integrator SI over the initial integration period, but both have errors that grow linearly, which is not the case of the symplectic integrator (some jumps in the error of the energy are observed at each close approach, but this error oscillates at a larger scale and is bounded above). The adaptive symplectic method outperforms all of them, having low errors initially which hardly grow at all during the integration. More details of this example, together with other calculations, are given in section 4 .

1.5. Summary. The remainder of this paper is set out as follows. The class of (scale invariant) Hamiltonian problems to be studied is presented in section 2. In section 3 we derive an optimal scaling function for those problems which are exactly scaling invariant. In section 4 we consider problems with one degree of freedom which may be approximately scaling invariant. Using backward error analysis we determine the optimal rescaling of the problem leading to the lowest energy error for a given 
computational cost. Section 5 reviews the LC and KS transformations in two and three dimensions, respectively. This is generalized to the Lennard-Jones potential, making the resulting Hamiltonian system scaling invariant and separable. The new method is numerically tested in section 6 and the effects of scaling determined in this case.

\section{Adaptive and symplectic methods for Hamiltonian problems.}

2.1. The Sundman and Poincaré regularizations. Let us consider the Hamiltonian

$$
H=T(\mathbf{q}, \mathbf{p})+V(\mathbf{q})=\frac{1}{2} \mathbf{p}^{T} M(\mathbf{q}) \mathbf{p}+V(\mathbf{q})
$$

with $\mathbf{q}=\left(q_{1}, \ldots, q_{k}\right)^{T}, \mathbf{p}=\left(p_{1}, \ldots, p_{k}\right)^{T}$, and $M$ a diagonal matrix, which at this stage we consider the identity $M=I$. A standard a priori adaptive technique for an ODE integration method is based on a time regularization so that the solution evolves on a fictive time $\tau$ used for all computations. Ideally, rapid variations in $t$ should correspond to moderate variations in $\tau$. This fictive time is introduced through the ordinary differential equation defining a Sundman transformation [25]

$$
\frac{d t}{d \tau}=g(\mathbf{q}, \mathbf{p})
$$

Here $g$ is a positive scalar function taken to be small if the solution is evolving rapidly. For this section we consider the scaling function $g$ to be arbitrary. In sections 3 and 4 we show how considerations of scale invariance allow it to be determined. This transformation leads to the system

$$
\frac{d \mathbf{p}}{d \tau}=-g \nabla_{\mathbf{q}} H=-g \nabla_{\mathbf{q}} V, \quad \frac{d \mathbf{q}}{d \tau}=g \nabla_{\mathbf{p}} H=g \mathbf{p} .
$$

In general, this system is no longer Hamiltonian. To recover a Hamiltonian structure we introduce two new conjugate coordinates, ${ }^{1} q^{t}=H\left(\mathbf{q}_{0}, \mathbf{p}_{0}\right)$ and $p^{t}=t$, which satisfy the differential equations

$$
\frac{d q^{t}}{d \tau}=0 \quad \text { and } \quad \frac{d p^{t}}{d \tau}=g
$$

The whole system $\left(\mathbf{q}, q^{t}, \mathbf{p}, p^{t}\right)$ then evolves in the fictive time, $\tau$, and is Hamiltonian with Hamiltonian function

$$
K=g(\mathbf{q}, \mathbf{p})\left(H(\mathbf{q}, \mathbf{p})-q^{t}\right) .
$$

$K$ remains constant at its initial value of 0 throughout time. As $g$ is positive, this implies that $H(q, p)-q^{t}=H(q, p)-H\left(q_{0}, p_{0}\right)$ also remains zero. Therefore, the original Hamiltonian is conserved. The extended system of ordinary differential equations that we now require to be solved is

$$
\begin{array}{ll}
\frac{d \mathbf{q}}{d \tau}=g \mathbf{p}+\nabla_{\mathbf{p}} g\left(H-q^{t}\right), & \frac{d q^{t}}{d \tau}=0, \\
\frac{d \mathbf{p}}{d \tau}=-g \nabla_{\mathbf{q}} V-\nabla_{\mathbf{q}} g\left(H-q^{t}\right), & \frac{d p^{t}}{d \tau}=g .
\end{array}
$$

\footnotetext{
${ }^{1}$ In the literature it is usual to take $q^{t}=t$ and $p^{t}=-H\left(\mathbf{q}_{0}, \mathbf{p}_{0}\right)$, but to get a more natural splitting of the extended Hamiltonian, in this paper we prefer to consider $p^{t}$ as the time.
} 
This system is a Poincaré transformation of the original [19, 35, 42]. We know that $H-q^{t}=0$ for the exact solution, but this is not necessarily the case for the numerical integrators, and for this reason this term is not removed in (2.5).

2.2. Hamiltonian problem and symplectic methods. Let us consider (2.1) with $M(\mathbf{q})=g(\mathbf{q}) I$, which has the same structure as (2.4) if $g$ depends only on coordinates. If $\hat{H}$ is the Lie operator associated to the Hamiltonian ${ }^{2}$ and $t$ is the time, the Lie transformation, $e^{-t \hat{H}}$, acting on $(\mathbf{q}, \mathbf{p})$ gives the evolution, which is a canonical or symplectic transformation. Suppose that $T$ and $V$ are both exactly solvable; the second order explicit, symmetric, and symplectic Störmer/leapfrog/Verlet method, for one time step $h$, is

$$
S_{2}(h)=e^{-\frac{h}{2} \hat{V}} e^{-h \hat{T}} e^{-\frac{h}{2} \hat{V}}=e^{-h \hat{H}}+O\left(h^{3}\right)
$$

giving the algorithm

$$
\begin{aligned}
\mathbf{p}_{n+1 / 2} & =\mathbf{p}_{n}-\frac{h}{2} \nabla_{\mathbf{q}} V\left(\mathbf{q}_{n}\right), \\
\left(\mathbf{q}_{n+1}, \overline{\mathbf{p}}_{n+1 / 2}\right) & =\Phi_{T}^{h}\left(\mathbf{q}_{n}, \mathbf{p}_{n+1 / 2}\right), \\
\mathbf{p}_{n+1} & =\overline{\mathbf{p}}_{n+1 / 2}-\frac{h}{2} \nabla_{\mathbf{q}} V\left(\mathbf{q}_{n+1}\right),
\end{aligned}
$$

where $\left(\mathbf{q}_{n}, \mathbf{p}_{n}\right) \simeq\left(\mathbf{q}\left(t_{n}\right), \mathbf{p}\left(t_{n}\right)\right)$ with $t_{n}=t_{0}+n h$, and $\Phi_{T}^{h}=e^{-h \hat{T}}$ corresponds to the flow associated to $T$. Suppose now that $T$ is not exactly solvable, but can be approximated by an $n$ th-order integrator, $\Psi_{T}^{h}=\Phi_{T}^{h}+O\left(h^{n+1}\right)$. If $\Psi_{T}^{h}$ is a symmetric, symplectic, and/or of second order, then these properties are also preserved by $S_{2}(h)$.

For $N$ steps, the last computation of $\nabla_{\mathbf{q}} V\left(\mathbf{q}_{n+1}\right)$ at each step can be reused in the next step, and only one evaluation of $\nabla_{\mathbf{q}} V$ per step is required. Fourth order symplectic methods can be obtained by composition of this method [40, 44]. For example, a well-known symmetric fourth order method is given by

$$
S_{4}(h)=S_{2}\left(x_{1} h\right) S_{2}\left(x_{0} h\right) S_{2}\left(x_{1} h\right)
$$

with $x_{1}=1 /\left(2-2^{1 / 3}\right), x_{0}=1-2 x_{1}$. Higher order methods are also presented in [44]. These methods have become very well known due to their good behavior for long time integration and recently, a number of new splitting and composition methods have appeared which are more efficient [30]. For Hamiltonians like (2.1), which are quadratic in momenta, we have that $\{V,\{V, T\}\}$ depends only on the coordinates and $\{V,\{V,\{V, T\}\}\}=0$. Then, if $e^{-h \hat{T}}$ is computed exactly or approximated with high accuracy, more efficient symplectic RKN composition methods like

$$
\Phi_{h}=\prod_{i=0}^{k} e^{-a_{i} h \hat{T}} e^{-b_{i} h \hat{V}}
$$

with appropriate coefficients $a_{i}, b_{i}$ can be used. In addition, if in (2.9), instead of the Lie operator associated to $b_{i} h V$ we consider the Lie operator associated to the most general potential $b_{i} h V+c_{i} h^{3}\{V,\{V, T\}\}$, the efficiency of the integrators can be further improved. To illustrate the benefits of using RKN methods, in this paper

\footnotetext{
${ }^{2}$ It is defined by its action on a differentiable function, $f(\mathbf{q}, \mathbf{p})$, as $\hat{H} f=\{H, f\},\{\cdot, \cdot\}$ being the usual Poisson bracket.
} 
we will consider the six-stage fourth order integrator $\left(R K N_{4}\right)$ and the 11-stage sixth order integrator $\left(R K N_{6}\right)$ presented in [6], both taking the form (2.9). Methods up to order eight, using the processing technique and the previous general potential, are presented in $[3,5]$.

2.3. Canonical transformations. We consider the case in which $g$ depends only on the coordinates $g \equiv g(\mathbf{q})$. This is a natural condition for the central force examples that we consider later in this paper, and at the same time the system simplifies slightly. Then the Hamiltonian (2.4) can be written as

$$
K=T(\mathbf{q}, \mathbf{p})+V\left(\mathbf{q}, q^{t}\right)=g(\mathbf{q}) \frac{1}{2} \mathbf{p}^{T} \mathbf{p}+g(\mathbf{q})\left(V(\mathbf{q})-q^{t}\right) .
$$

The evolution due to the Hamiltonian $T=g(\mathbf{q}) \mathbf{p}^{T} \mathbf{p} / 2$ can be very efficiently approximated using implicit symplectic integrators because of its particular structure [19, $13,12]$. Then $S_{2}(h)$ in $(2.6)$ is also symmetric and, by composition, higher orders can be obtained. However, if we proceed this way, the more efficient RKN methods cannot be used. For this reason, we believe it is interesting to study for which functions $g$ the evolution of $T$ is exactly solvable. This can be considered equivalent to finding a set of coordinates $(\mathbf{Q}, \mathbf{P})$ derived from those used in the Poincaré transformation, so that the Hamiltonian (2.10), in the transformed coordinates, has a separable form like $H$. We can then apply a splitting Nyström method, such as given in section 2.2, to this new system.

Describing the evolution of a system using Hamiltonian equations has the advantage that $\mathbf{q}$ and $\mathbf{p}$ are independent coordinates which play symmetric roles in the equations. This allows us to choose the most appropriate coordinates and momenta to describe a particular system. In the numerical integration of separable systems, it is important to take into account the cost of the computation associated to each part of the Hamiltonian. If new coordinates and momenta $\mathbf{Q}, \mathbf{P}$ are found, giving a Hamiltonian that is simpler and faster to compute with, then the performance of the integrators can improve. If the transformation $(\mathbf{q}, \mathbf{p})=\mathcal{C}(\mathbf{Q}, \mathbf{P})$ is canonical, the Hamiltonian which describes the evolution on the new coordinates is $H(\mathbf{Q}, \mathbf{P})=H(\mathbf{q}(\mathbf{Q}, \mathbf{P}), \mathbf{p}(\mathbf{Q}, \mathbf{P}))$. The problem we then face is to find the canonical transformations (CT's) which transform a complicated Hamiltonian into a simpler one, for example, one which changes a nonseparable Hamiltonian into a separable one. For this purpose, the following theorem is very useful [18, 27].

THEOREM 2.1. The transformation

$$
\left\{\begin{aligned}
\mathbf{q} & =\Phi(\mathbf{Q}) \\
\Phi^{\prime}(\mathbf{Q})^{T} \mathbf{p} & =\mathbf{P}
\end{aligned}\right.
$$

where $\Phi$ is a diffeomorphism of $\mathbb{R}^{2 k}$, is canonical.

2.3.1. Example. In order to illustrate the benefits of using a CT for the numerical integration of a Hamiltonian system, let us consider the Hamiltonian (1.1) with the regularization function $g=q^{3 / 2}$. The motivation for this particular choice will come later when we consider scaling symmetries. Now, (2.10) takes the form

$$
K=T(q, p)+V\left(q, q^{t}\right)=\frac{1}{2} q^{3 / 2} p^{2}+\left(-q^{1 / 2}+\frac{\varepsilon}{q^{1 / 2}}-q^{t} q^{3 / 2}\right),
$$

where both $T$ and $V$ are exactly solvable (the Hamiltonian $H=q^{\alpha} p^{\beta}$, with $\alpha, \beta$ constants, is exactly solvable). However, $T$ can be relatively expensive to compute, 
and can accumulate large errors if $q$ is small and $p$ large. If the same problem is considered using new coordinates $(Q, P)$, related to the old coordinates through the $\mathrm{CT}$

$$
q=Q^{4}, \quad p=P /\left(4 Q^{3}\right), \quad p^{t}=P^{t}, \quad q^{t}=Q^{t},
$$

the Hamiltonian to be solved is

$$
K=\frac{1}{32} P^{2}+\left(-Q^{2}+\frac{\varepsilon}{Q^{2}}-Q^{t} Q^{6}\right) .
$$

If a splitting method is used in (2.12), at least one square root per stage is needed. However, if the same splitting method is applied to (2.13) no square root is required and a smaller number of arithmetic operations is also required. The only root needed is for starting the computation. In addition, as mentioned above, near a collision the computation of $q^{3 / 2} p^{2}$ can be problematic in the accumulation of roundoff errors, and this is avoided using (2.13). If at a given instant the output is desired in the old coordinates, this can also be cheaply computed.

2.4. Other explicit adaptive integrators. In the following we present some of the most important explicit adaptive integrators for Hamiltonian systems.

2.4.1. Symmetric nonsymplectic methods. Most Hamiltonian systems are reversible, and symmetric linear one-step methods preserve this symmetry [39], and usually show qualitative properties similar to symplectic integrators. The following second order symmetric nonsymplectic adaptive Verlet method (adapted from the symmetric and symplectic velocity Verlet algorithm $(2.7))$ is presented in $[22,7,21]$ :

$$
\begin{aligned}
\mathbf{p}_{n+1 / 2} & =\mathbf{p}_{n}-\frac{h}{2 \rho_{n}} \nabla_{\mathbf{q}} V\left(\mathbf{q}_{n}\right), \\
\mathbf{q}_{n+1 / 2} & =\mathbf{q}_{n}+\frac{h}{2 \rho_{n}} \mathbf{p}_{n+1 / 2}, \\
\rho_{n+1}+\rho_{n} & =2 / g\left(\mathbf{q}_{n+1 / 2}, \mathbf{p}_{n+1 / 2}\right), \\
\mathbf{q}_{n+1} & =\mathbf{q}_{n+1 / 2}+\frac{h}{2 \rho_{n+1}} \mathbf{p}_{n+1 / 2}, \\
\mathbf{p}_{n+1} & =\mathbf{p}_{n+1 / 2}-\frac{h}{2 \rho_{n+1}} \nabla_{\mathbf{q}} V\left(\mathbf{q}_{n+1}\right) .
\end{aligned}
$$

To start the integration one can use $\rho_{0}=1 / g\left(x_{0}\right)$ but this choice might lead to oscillations in the numerically computed $\rho_{n}$ 's, which can be avoided choosing a modified initialization [15]. This can be considered as the basic integrator, $S_{2}(h)$, to build higher order methods by composition. We must also mention that Nyström methods for problems with quadratic kinetic energy cannot be used. In addition, choosing the previous value for $\rho_{0}$ introduces new error terms in the algorithm (backward error analysis [15]) which cannot be canceled by composition to get methods of order higher than four. This problem can be trivially avoided choosing as the new basic method, $S_{2}(h)$, two steps of (2.14) [41].

2.4.2. Generalized logarithmic Hamiltonian methods. Suppose that for solving the Hamiltonian problem (2.5) we consider [34]

$$
g=\frac{f\left(T(\mathbf{p})-q^{t}\right)-f(-V(\mathbf{q}))}{H(\mathbf{q}, \mathbf{p})-q^{t}}
$$



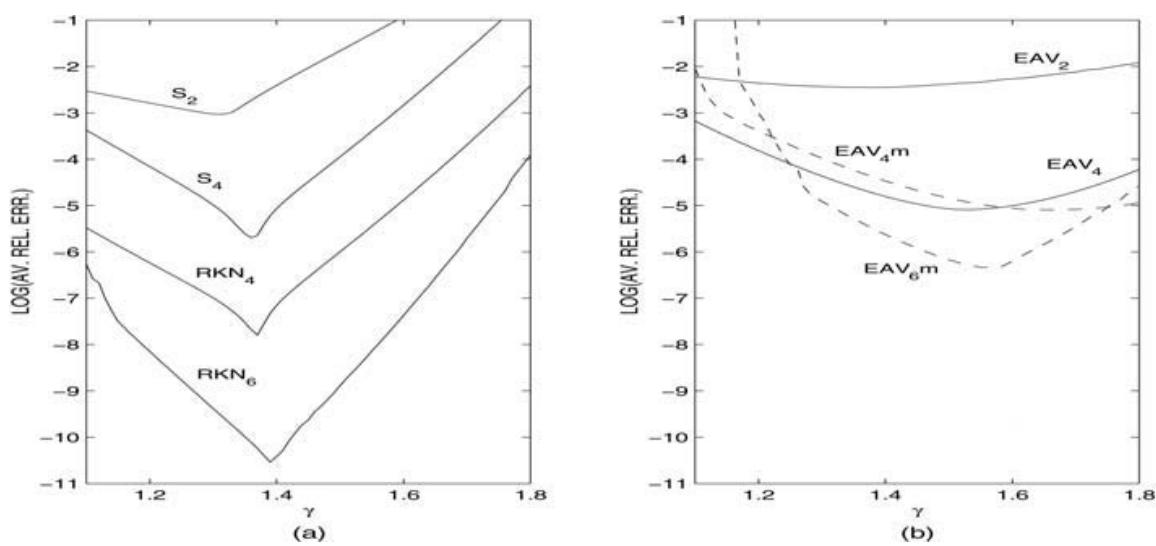

FIG. 3.1. Average error in energy in logarithmic scale in the integration of (1.1). The system is integrated until the final real time $t=20$. All integrations require, approximately, the same number of potential evaluations for each value of $\gamma$.

with $f(u)$ an analytic function. Observe that the apparent singularity at $H=q^{t}$ is removable (take the Taylor series of $f$ about the point $-V(\mathbf{q})$ and use $T(\mathbf{p})-q^{t}=$ $\left.H(\mathbf{q}, \mathbf{p})-q^{t}-V(\mathbf{q})\right)$. Since numerically $T(\mathbf{p})-q^{t} \simeq-V(\mathbf{q})$, then $g \simeq f^{\prime}(-V(\mathbf{q}))$, where $f(u)$ has to be a function such that $f^{\prime}(u)>0$, with no singularities in the region of interest. Substituting into (2.4), the Hamiltonian to solve is

$$
K=f\left(T(\mathbf{p})-q^{t}\right)-f(-V(\mathbf{q}))
$$

which is again separable, but RKN methods cannot be used. The particular case $f(u)=\log (u)$ has been considered in [31, 32].

3. Choice of the monitor function $g(u)$. The performance of the numerical integrator described above is often highly dependent on the function $g$, and as we will show, this dependence drastically increases with the order of the integrator. For this reason, it is very important to have a simple criterion to find a good regularization function for a given problem. This criterion seems to be closely related to the scaling invariance of the dominant part of the potential in those regions where the system evolves rapidly. We see this by looking at a particular example.

Example. We consider the Hamiltonian (1.1) with $\varepsilon=0.001$ and integrate the system using a regularization function

$$
g(q)=q^{\gamma}
$$

(the reason for this choice will be made clear in section 3.2) for different values of $\gamma$. We take all integrations until $t=20$. The trajectories of this problem are periodic orbits with close approaches to the origin, where the particle moves rapidly and adaptivity is necessary. To compare methods we measure the average error in the energy (Hamiltonian) using a time step, $\Delta \tau$, such that the final time is always reached with approximately 12000 force evaluations. Figure 3.1a shows the results obtained with splitting RKN integrators, which solve the transformed kinetic energy $T=\frac{1}{2} q^{\gamma} p^{2}$ exactly so that the formulae (2.6)-(2.9) can be used. In particular we consider the second order, $S_{2}$, and the fourth order, $S_{4}$, methods given in (2.6) and (2.8), respectively, as well as $R K N_{4}$ and $R K N_{6}$. We can observe very sharp minima (notice the 
logarithmic scale of the error) with a clear dependence on the order of the method. We repeat the same experiment taking the adaptive Verlet method (2.14) as the basic integrator $S_{2}$ (EAV2), and EAV4 corresponds to the fourth order 5 stage composition given in [40] (solid lines). To get higher order methods we take as $S_{2}$ two steps of (2.14) (EAV4m and EAV6m). The sixth order method EAV6m uses the symmetric composition with nine steps of $S_{2}$ given in [28]. Figure 3.1b shows the results obtained, and we can also observe that for both families of methods (broken and solid lines) the curves turn sharper with the order. It is clear that to obtain accurate results it is very important to choose $\gamma$ carefully. Comparing the results of $S_{4}$ and $R K N_{4}$ in the left figure, we can also appreciate the benefits of using a Nyström method versus a more general integrator. To compare the performance between methods in Figures 3.1a and $3.1 \mathrm{~b}$, it is necessary to consider the values of $\gamma$ chosen as well as the computational cost, and this is highly problem dependent. If the cost for the computation of the evolution of $T$ is expensive, the EAV methods can be superior for a range of values of $\gamma$.

The optimal value of $\gamma$ as seen in these graphs is quite different from $\gamma=0$ (constant time step), $\gamma=1$ (the Levi-Civita scaling), or $\gamma=2$ (constant angle), but lies close to the scale invariant value of $\gamma=1.5$. In addition, we observe that for each family of methods, the minimum approaches this value when increasing the order. In section 4 we return to give a more detailed analysis of this example using backward error analysis for the splitting and symplectic methods, and show that $\gamma \rightarrow 1.5$ as the order of the method increases. We now consider two systematic approaches for finding $g$.

3.1. Computational cost and backward error analysis. A reasonable condition on the choice of $g$ is that the average error in the computation of the Hamiltonian should be minimized for a given computational cost. An application of backward error analysis [20] implies that a symplectic integrator for a Hamiltonian system with Hamiltonian $K$ used with a fixed time step, $\Delta \tau$, solves a discrete system which corresponds exactly (up to exponentially small terms in $\Delta \tau$ ) to the solutions of a perturbed Hamiltonian system $K+\Delta \tau K_{2}+\Delta \tau^{2} K_{3}+\cdots$. If the integrator is a splitting method (with the evolution due to $T$ exactly computed), the functions $K_{i}$ are linear combinations of nested commutators of the parts of the Hamiltonian [44]. In particular we have that

$$
K_{2}=k_{21}\{T, V\}, \quad K_{3}=k_{31}\{V,\{V, T\}\}+k_{32}\{T,\{V, T\}\} \ldots,
$$

where the coefficients $k_{i j}$ depend only on the particular integrator used. For instance, $k_{i j}=0, i=2, \ldots, n, j=1, \ldots$ if the integrator is of order $n$. For simplicity, let us consider the one-dimensional problem with $T=\frac{1}{2} g(q) p^{2}$ and $V=g(q)\left(\tilde{V}(q)-q^{t}\right)$, then

$$
K_{2}=k_{21} g V^{\prime} p, \quad K_{3}=k_{31} g\left(V^{\prime}\right)^{2}-\frac{k_{32}}{2} g\left(V^{\prime} g^{\prime}+2 g V^{\prime \prime}\right) p^{2}, \ldots
$$

To obtain a realistic error analysis we look at the error in the unscaled Hamiltonian $H$, where, up to a constant, $H=K / g$. Thus, $H$ is perturbed to $H+\Delta \tau H_{2}+\Delta \tau^{2} H_{3}+\cdots$ where $H_{i}=K_{i} / g$. We aim to choose the scaling function $g$ for an $n$ th-order method so that the average of $\Delta \tau^{n} H_{n+1}$ over an orbit is minimized for a fixed computational cost. In general, this calculation will be very hard, and more detailed information on the problem is needed to find the optimal $g$. However, for certain problems with a scaling structure we can make estimates for the size of each term to guide the choice of $g$. This we do in section 4.3 . 
3.2. Scale invariance. A second motivation of our choice of the function $g$ is the phenomenon of scale invariance. Adaptive methods for scale invariant problems, which themselves inherit the invariance, shadow self-similar solutions with a uniform error and inherit any conservation properties of the original problem which are linked to symmetries. Moreover, it is relatively easy to identify functions $g(\mathbf{q})$ which lead to scale invariant discretizations. Suppose that we consider the (not necessarily Hamiltonian) ODE system $d u_{i} / d t=f_{i}\left(u_{1}, \ldots, u_{N}\right), i=1, \ldots, N$. This system is scale invariant if it remains unchanged under the transformation

$$
t \rightarrow \lambda t, \quad u_{i} \rightarrow \lambda^{\alpha_{i}} u_{i}
$$

where $\lambda>0$ is an arbitrary real number and the $\alpha_{i}$ depend on the problem. A solution of such a system is self-similar if $u_{i}(\lambda t)=\lambda^{\alpha_{i}} u_{i}(t)$. Such solutions have the general form $u_{i}=t^{\alpha_{i}} v_{i}$, where the constants $v_{i}$ satisfy an algebraic equation. The Kepler problem admits (both regular and singular) self-similar solutions with zero angular momentum, as well as nonself-similar periodic solutions with nonzero angular momentum. Applying the Sundman transformation to this system, we have

$$
d t / d \tau=g(\mathbf{u}) \quad \text { and } \quad d u_{i} / d \tau=g(\mathbf{u}) f_{i}(\mathbf{u}) .
$$

Unless $g$ is chosen appropriately, this system is no longer scale invariant. However, with a careful choice of $g$ we can construct a system which has the desirable property that two solutions mapped into each other by the scaling relation (3.1) evolve at the same fictive time. This is a desirable property for an adaptive method which can use a constant step size $\Delta \tau$ for two solutions evolving on different spatial scales. To achieve scale invariance the function $g$ must satisfy the functional equation

$$
g\left(\lambda^{\alpha_{1}} u_{1}, \lambda^{\alpha_{2}} u_{2}, \ldots, \lambda^{\alpha_{N}} u_{N}\right)=\lambda g\left(u_{1}, u_{2}, \ldots, u_{N}\right) .
$$

In general, functions $g(u)$ which satisfy such scaling laws are either multi-variable polynomials or are functions of groupings of the variables $u_{i}$ which scale in a similar manner. For the two-dimensional Kepler problem $\left(V=-1 / r\right.$ with $r=\left(q_{1}^{2}+q_{2}^{2}\right)^{1 / 2}$ in (2.1)) we have $g(\mathbf{q})=r^{3 / 2}$.

Suppose that we now discretize the transformed system with a multi-step or Runge-Kutta method with a constant step size $\Delta \tau$, so that $U_{i}^{n} \approx u_{i}(n \Delta \tau)$ is a discrete approximation of $u(\tau)$ at time $t_{n}$. If the underlying ODE admits a self-similar solution then the following result is proved in [8].

THEOREM 3.1. (i) If $\Delta \tau$ is sufficiently small, then the discrete problem admits a discrete self-similar solution of the form $U_{i}^{n}=z^{\alpha_{i} n} V_{i}, t_{n}=z^{n}$;

(ii) if $u(t)$ is the true self-similar solution, then $\left\|U_{i}^{n}-u_{i}\left(t_{n}\right)\right\|=u_{i}\left(t_{n}\right)(1+$ $\left.\mathcal{O}\left(\Delta \tau^{p}\right)\right)$, where the implied constant in the order relationship does not depend upon $n$;

(iii) if the true self-similar solution is an attractor, then so is the discrete selfsimilar solution.

This theorem applies only for problems which are scale invariant. In practice most problems arising in applications are not truly scale invariant, but are approximately so (for example, close to a collision). This is true for the Hamiltonian (1.1) when $\varepsilon \neq 0$. In this case the choice of $g(u)$ may not be optimal, but should still give reasonable results, and we explore this in the next section. 
4. Regularization and error analysis for problems with one degree of freedom.

4.1. Preserving scaling invariance for the one-dimensional problem. Suppose that the Hamiltonian equations obtained from (2.1) are invariant under a linear scaling

$$
(t, q, p) \rightarrow\left(\lambda t, \lambda^{\alpha_{1}} q, \lambda^{\alpha_{2}} p\right)
$$

This then leads to certain restrictions on the scaling function $g(q)$. We consider this question in the context of a central force type of equation. Suppose the potential is given by $V(q)=C / q^{r}$. Then the Hamiltonian equations are invariant when

$$
\left(\alpha_{1}, \alpha_{2}\right)=\left(\frac{2}{2+r}, \frac{-r}{2+r}\right) .
$$

Consider that the scaling type regularization function, $g(q)=q^{\gamma}$, has been introduced and the phase space enlarged with $\left(q^{t}, p^{t}\right)$. The Hamiltonian (2.10) for the extended system is now

$$
K\left(p, q, p^{t}, q^{t}\right)=\frac{1}{2} g(q) p^{2}+g(q)\left(V(q)-q^{t}\right)=\frac{1}{2} q^{\gamma} p^{2}+C q^{\gamma-r}-q^{t} q^{\gamma},
$$

and the corresponding differential equations are

$$
\begin{array}{lll}
\frac{d q}{d \tau}=q^{\gamma} p, & \frac{d q^{t}}{d \tau}=0, \\
\frac{d p}{d \tau}=-\frac{\gamma}{2} p^{2} q^{\gamma-1}-(\gamma-r) C q^{\gamma-r-1}+\gamma q^{t} q^{\gamma-1}, & \frac{d p^{t}}{d \tau}=q^{\gamma} .
\end{array}
$$

Now consider the scalings of this transformed equation of the form $\left(q, q^{t}, p, p^{t}, \tau\right) \rightarrow$ $\left(\lambda^{\beta_{1}} q, \lambda^{\beta_{2}} q^{t}, \lambda^{\beta_{3}} p, \lambda^{\beta_{4}} p^{t}, \lambda^{\beta_{5}} \tau\right)$. As the original system is scale invariant it is important that the transformed system should also have this property. Furthermore, we require that all solutions, at all scales, should evolve with the same fictive time, so that $\beta_{5}=0$. This is the phenomenon of scaling invariance described in section 3.2, i.e., the numerical method should perform equally well at all scales. Inspection of the equation for $d q / d \tau$ shows that $\beta_{3}=(1-\gamma) \beta_{1}$. Making this substitution in the equation for $d p / d \tau$ implies that we have scale invariance only if

$$
\gamma=\gamma_{s c} \equiv 1+r / 2
$$

so that $\gamma_{s c}=3 / 2$ if $r=1$.

4.2. Example. We consider again the one-dimensional Hamiltonian

$$
H=\frac{1}{2} p^{2}-\frac{1}{q^{r}}+\frac{\varepsilon}{q^{s}}
$$

with $s>r$ and $\varepsilon \geq 0$. If $H<0$ and $\varepsilon>0$, then the solution is bounded and periodic, and it has very close approaches to the origin when $\varepsilon \ll 1$. If $\varepsilon=0$ and $H \leq 0$, then $q \rightarrow 0$ and $|p| \rightarrow \infty$ in a finite time $T$. In either case the solution evolves on small time scales and, hence, small time steps must be used for these parts of the motion, whilst longer time steps are desirable when $q$ is not small. Taking $g=q^{\gamma}$, the Hamiltonian to be solved becomes

$$
K=\frac{1}{2} q^{\gamma} p^{2}-q^{\gamma-r}+\varepsilon q^{\gamma-s}-q^{t} q^{\gamma}
$$


When $\varepsilon=0$ the differential equations derived from this Hamiltonian are exactly invariant under the change of scale given by (4.1) and (4.2). There is an exact (singular) self-similar solution of the form

$$
q(t)=C(T-t)^{\alpha}, \quad p(t)=C \alpha(T-t)^{\alpha-1},
$$

where $\alpha=2 /(r+2), C=\left(2 / \alpha^{2}\right)^{1 /(r+2)}$, which acts as an attractor [8]. Here $T$ is a finite time at which the singularity occurs. In this case a scale invariant numerical method with $\gamma=\gamma_{s c}$ is a natural choice for integrating the differential equation.

If $\varepsilon>0$, then the differential equations are not precisely invariant under this change of scale, but are close to being so provided that $q>\varepsilon^{1 /(s-r)}$, and in this case the corresponding solutions are close to the self-similar solution.

4.3. Error analysis. We now combine the backward error analysis of section 3.1 with the scaling ideas to give an explanation of the distinctive form of the graphs in Figure 3.1a, and to estimate the average energy error given a fixed computational cost for the more general Hamiltonian (4.6).

Suppose that we consider a periodic orbit with initial values of $(q, p)=(1,0)$ so that $H=\varepsilon-1$. If $\varepsilon>0$ is small, then the orbit has a close approach so that the minimum value of $q$ is given by $q_{\min } \approx \varepsilon^{1 /(s-r)}$ with $d^{2} q / d t^{2} \approx 1 / \varepsilon^{(r+1) /(s-r)}$ at this point. Similarly, the maximum value of $p$ is given by $p_{\max } \approx \varepsilon^{-r / 2(s-r)}$ and occurs when $q=q^{*}=(s \varepsilon / r)^{1 /(s-r)}$. Thus this orbit is close to being singular at this point. We identify two regions of the orbit, the outer region in which $q>q^{*}$ and $|p|$ increases to $p_{\max }$ and an inner region in which $q_{\min }<q<q_{\max }$ and $0<|p|<p_{\max }$. A simple estimate, using the approximation of $d^{2} q / d t^{2}$, implies that the time scale $T_{2}$ of the inner region is proportional to

$$
T_{2}=C(s, r) \varepsilon^{(r+2) / 2(s-r)}, \quad \text { with } C(s, r)=\left(\frac{\left(\frac{s}{r}\right)^{1 /(s-r)}-1}{s-r}\right)^{1 / 2}
$$

but for simplicity we will consider $T_{2}=\varepsilon^{(r+2) / 2(s-r)}$. Using the scaling function $g=q^{\gamma}$, the time step $\Delta t$ selected for this part of the motion (over which the scaling symmetry of the problem does not apply, as $1 / q^{r}$ is comparable to $\left.\varepsilon / q^{s}\right)$ is approximately uniform at $\Delta t \approx \varepsilon^{\gamma /(s-r)} \Delta \tau$. The outer region exists for times increasing from zero until the time $T-T_{2}$ close to the collapse time $T$ for the unperturbed problem. The equation is close to being scaling invariant in the outer region, and the solution lies close to the singular self-similar solution (4.8) for which $p^{2}=2 / q^{r}$. From the previous analysis we know that this self-similar solution is approximated optimally well if we use a scale invariant discretization in this region. If a monitor function of the form $g=q^{\gamma}$ is used, then the following heuristic argument explains the errors that we observe. If $\gamma$ is too small, then too large, a time step is chosen and the motion in the inner region is poorly approximated, leading to large errors. Conversely, if $\gamma$ is too large, then a small time step is chosen and a large number of calculations are made in the inner region. If the overall computational cost is to be held constant, then fewer calculations are made in the outer region and the error here is large. It is only when it is close to the scaling invariance value that we achieve the right balance between computational cost and accuracy. We now show that for the transformed symplectic methods the optimal value is

$$
\gamma \simeq \frac{r+2}{2}+\frac{r-2}{2 n}=\gamma_{s c}+\frac{r-2}{2 n},
$$



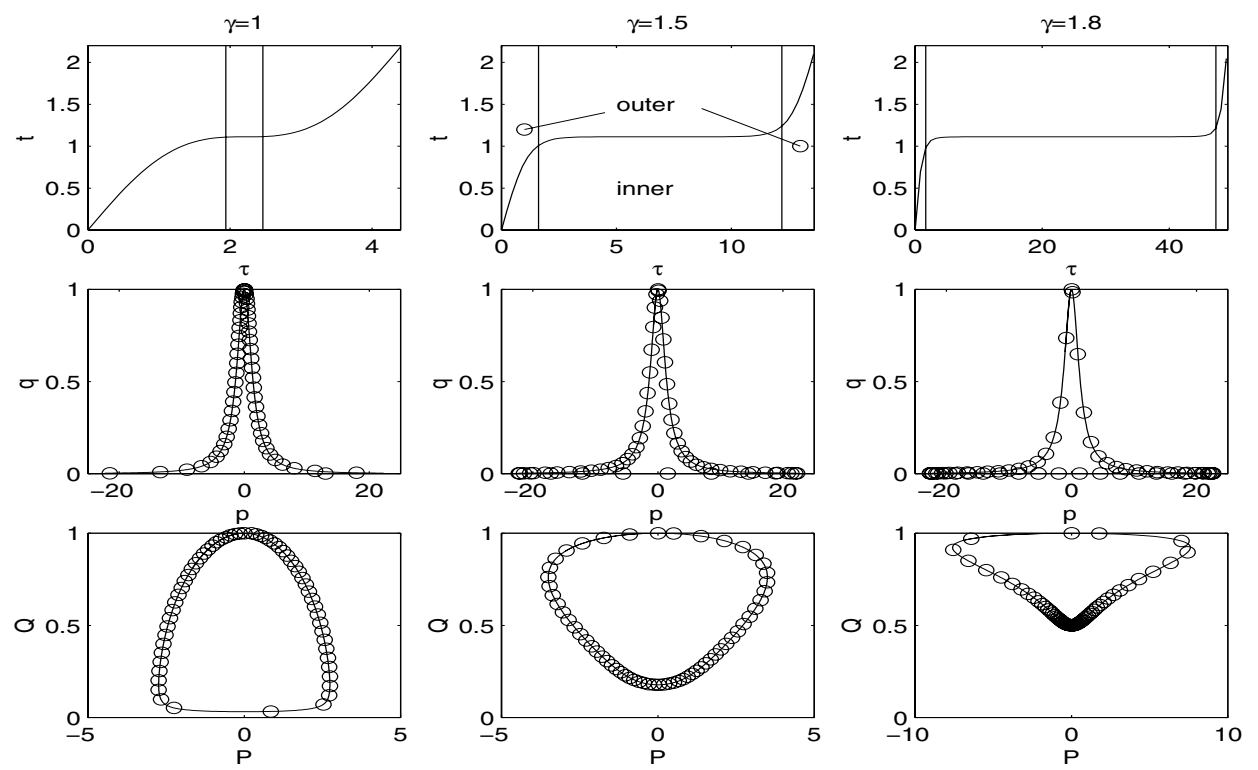

FIG. 4.1. Top figures: Plots of $t$ versus computational time $\tau$, for $r=1, s=2, \varepsilon=0.001$, $N=60$, and $\gamma=1,1.5,1.8$. The vertical lines separate the inner from the outer regions. Medium and bottom figures: Rescaled trajectories in the $q-p$ and $Q-P$ phase spaces.

where $n$ is the order of the method.

Computational cost. We presume that all calculations are made in the computational $\tau$ space and that $\Delta \tau$ is uniform, and we make $N$ calculations overall (with one computation per computation time-step). A computational time $\tau_{1}$ is spent in the outer region and a time $\tau_{2}$ in the inner region. This is illustrated by Figure 4.1, where we present how the true time varies as a function of the computational time for $r=1, s=2, \varepsilon=0.001$, and $\gamma=1,1.5,1.8$, taking $N=60$.

The true time $T_{2}$ spent in the inner region is proportional to $\varepsilon^{(r+2) / 2(s-r)}$ so that, as $g(q) \approx \varepsilon^{\gamma /(s-r)}$ in this region, we have

$$
\tau_{2}=\mathcal{O}\left(\varepsilon^{\left(1+\frac{r}{2}-\gamma\right) /(s-r)}\right)
$$

Note that $\tau_{2}$ is small if $\gamma<1+\frac{r}{2}$ and large if $\gamma>1+\frac{r}{2}$. In the outer region $q(t)$ approximates the self-similar solution which is proportional to $(T-t)^{2 /(2+r)}$ close to the point of singularity. We can make an order of magnitude estimate of $\tau_{1}$ by assuming that the true time interval for the outer region is given by $t \in[0, T-$ $\left.\varepsilon^{(1+r / 2) /(s-r)}\right]$. From the identity $d t / d \tau=g(u)$ we have that

$$
\tau_{1} \approx \int_{0}^{T-\varepsilon^{(1+r / 2) /(s-r)}}(T-t)^{-2 \gamma /(2+r)} d t= \begin{cases}\mathcal{O}(1) & \text { if } \gamma<1+\frac{r}{2} \\ \mathcal{O}(\log (\varepsilon)) & \text { if } \gamma=1+\frac{r}{2} \\ \mathcal{O}\left(\varepsilon^{\left(1+\frac{r}{2}-\gamma\right) /(s-r)}\right) & \text { if } \gamma>1+\frac{r}{2}\end{cases}
$$

As $N$ calculations are made in the computational time space, we have $\Delta \tau=\left(\tau_{1}+\right.$ 
$\left.\tau_{2}\right) / N$, so that

$$
\begin{array}{ll}
\Delta \tau=\mathcal{O}(1 / N) & \text { if } \gamma<1+\frac{r}{2}, \\
\Delta \tau=\mathcal{O}(\log (\varepsilon) / N) & \text { if } \gamma=1+\frac{r}{2}, \\
\Delta \tau=\mathcal{O}\left(\varepsilon^{\left(1+\frac{r}{2}-\gamma\right) /(s-r)} / N\right) & \text { if } \gamma>1+\frac{r}{2} .
\end{array}
$$

Backward error analysis. We can now apply the backward error analysis of section 3.1 by making estimates of the terms $H_{i}$ for the orbit we are considering. Here the scaling structure of the problem works strongly to our advantage as the various dominant commutator terms involved in $H_{i}$ all scale in a similar manner and the precise values of the coefficients $k_{i j}$ do not matter. In particular we have that $T=q^{\gamma} p^{2} / 2$ and $V=-q^{\gamma-r}+\varepsilon q^{\gamma-s}-q^{t} q^{\gamma}$. As we are interested in the dominant contributions to the error, expressions involving $q^{t} q^{\gamma}$ can be neglected so that the dominant terms in $H_{2}$ take the form $p q^{\gamma-r-1}, \varepsilon p q^{\gamma-s-1}$, the dominant terms in $H_{3}$ take the form $q^{2 \gamma-2 r-2}, p^{2} q^{2 \gamma-r-2}, \varepsilon q^{2 \gamma-r-s-2}, \varepsilon p^{2} q^{2 \gamma-s-2}$, and $\varepsilon^{2} q^{2 \gamma-2 s-2}$, etc. In the inner region, $p$ is bounded above by $\varepsilon^{-r /(2(s-r))}$ and $q$ is bounded below by $\varepsilon^{1 /(s-r)}$. Thus, all dominant terms in $H_{i}$ scale in the same way with $H_{2}$ bounded by $\varepsilon^{(\gamma-1-3 r / 2) /(s-r)}$, $H_{3}$ by $\varepsilon^{2(\gamma-1-2 r) /(s-r)}, H_{4}$ by $\varepsilon^{3(\gamma-1-5 r / 2) /(s-r)}$, etc. Continuing this calculation inductively, it follows that in the inner region

$$
H_{i+1}<C \varepsilon^{[i(\gamma-1-r / 2)-r] /(s-r)} .
$$

In the outer region we exploit the fact that $\varepsilon$ is small and that $(q, p)$ are close to the self-similar solution (4.8). Again, all terms scale similarly and we have

$$
H_{i+1} \sim(T-t)^{[i(\gamma-1-r / 2)-r] /(1+r / 2)} .
$$

Using (4.13) and (4.14) we may now determine the average contribution, $E$, to the error over one period for a method of order $n$. This is given by combining the estimate over the inner and outer regions so that

$$
\begin{aligned}
E= & \Delta \tau^{n} \int_{0}^{T} H_{n+1}(t) d t \simeq \Delta \tau^{n} \int_{T-\varepsilon^{(r+2) / 2(s-r)}}^{T} C \varepsilon^{\frac{1}{s-r}\left[n\left(\gamma-1-\frac{r}{2}\right)-r\right]} d t \\
& +\Delta \tau^{n} \int_{0}^{T-\varepsilon^{(r+2) / 2(s-r)}}(T-t)^{\left[n\left(\gamma-1-\frac{r}{2}\right)-r\right] /(1+r / 2)} d t
\end{aligned}
$$

and so

$$
E=C \varepsilon^{\frac{1}{s-r}\left[n\left(\gamma-1-\frac{r}{2}\right)+1-\frac{r}{2}\right]} \Delta \tau^{n}+ \begin{cases}\mathcal{O}\left(\varepsilon^{\frac{1}{s-r}\left[n\left(\gamma-1-\frac{r}{2}\right)+1-\frac{r}{2}\right]} \Delta \tau^{n}\right) & \text { if } \gamma<\gamma^{*}, \\ \mathcal{O}\left(\Delta \tau^{n}\right) & \text { if } \gamma>\gamma^{*}\end{cases}
$$

Here $\gamma^{*}$ is the critical exponent for which both $\left[n\left(\gamma-1-\frac{r}{2}\right)-r\right] /(1+r / 2)=-1$ and $\frac{1}{r}\left[n\left(\gamma-1-\frac{r}{2}\right)+1-\frac{r}{2}\right]=0$ so that $\gamma^{*}$ is given by (4.10).

The value of $\Delta \tau$ above depends upon $\gamma$ from the previous analysis, so that $\Delta \tau$ is $\mathcal{O}(1 / N)$ if $\gamma<1+r / 2$ and $\Delta \tau$ is $\mathcal{O}\left(\varepsilon^{\left(1+\frac{r}{2}-\gamma\right) /(s-r)} / N\right)$ if $\gamma>1+r / 2$. Substituting these estimates into (4.16) and extracting the dominant error contribution, we have that

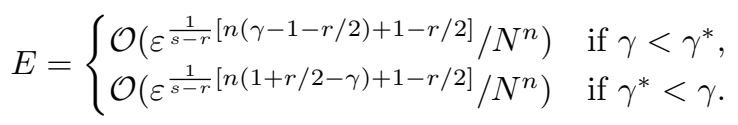



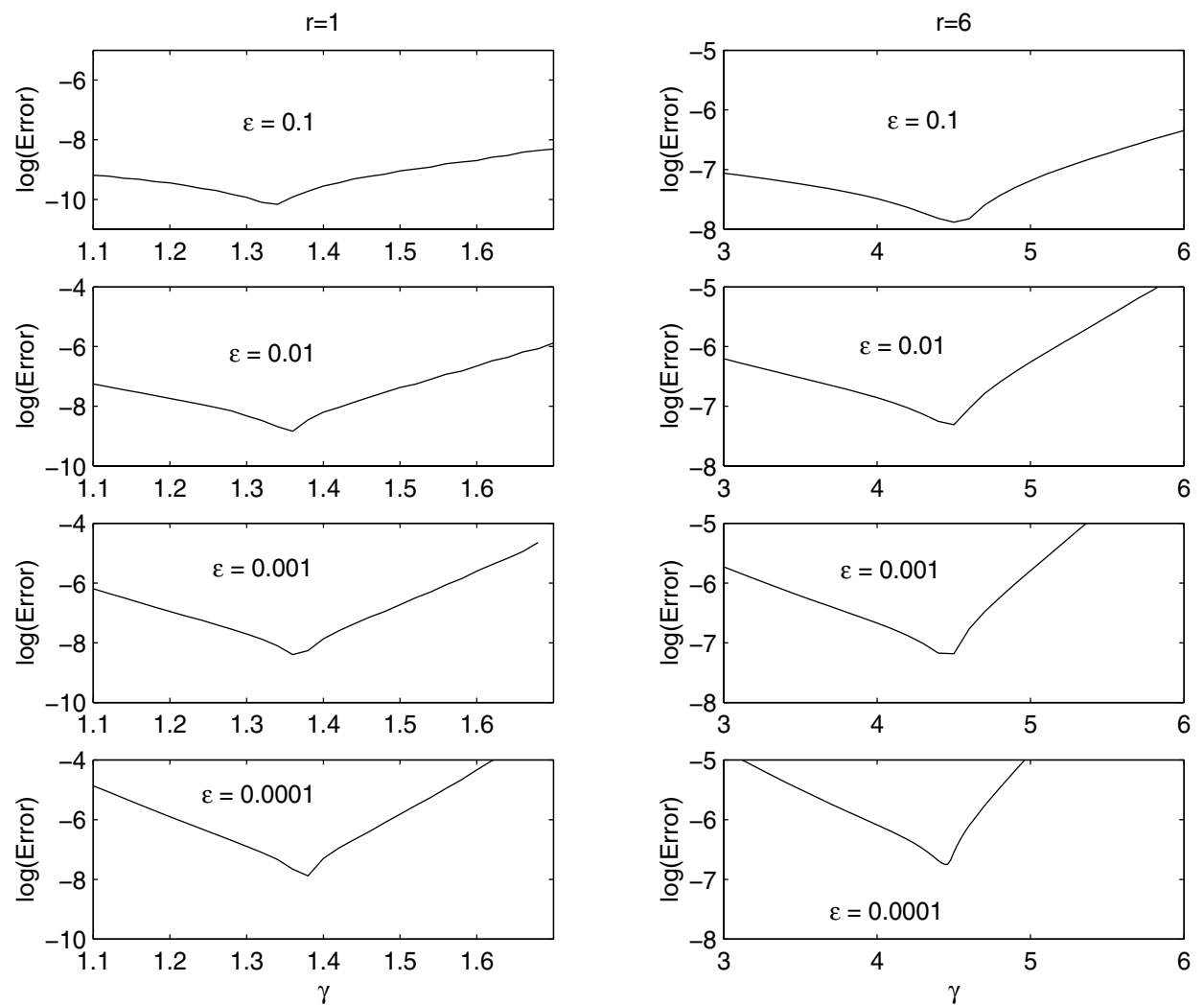

FIG. 4.2. Average error in energy in logarithmic scale in the integration of (4.6). The system is integrated until the final real time $t=20$ for $r=1, s=2$ (left column), and until $t=10$ for $r=6, s=12$ (right column). For each particular value of $\varepsilon$ all integrations require, approximately, the same number of force evaluations.

Thus $E$ has a minimum at $\gamma=\gamma^{*}$ and it is clear that $\gamma^{*}$ approaches the scaling invariant value of $1+\frac{r}{2}$ as $n \rightarrow \infty$.

Numerical experiments. In the limit $\varepsilon \rightarrow 0$ the evolution of the orbit becomes more nearly singular and we can study whether the choice (4.10), which nearly preserves scaling invariance in the fictive time, approaches the numerically observed optimal value. To find the optimal choice of $\gamma$ numerically, we consider problems with $\varepsilon=$ $0.1,0.01,0.001,0.0001$, and a range of values of $\gamma$, on the following two cases.

Case 1: The Kepler problem $(r=1, s=2)$. We integrated the system until $t=20$ for different values of $\gamma$ and measured the average error in energy using a time step, $\Delta \tau$, such that the final time, $t=20$, is reached in approximately $1000(\varepsilon=0.1)$, $1500(\varepsilon=0.01), 3000(\varepsilon=0.001)$, and $5000(\varepsilon=0.0001)$ steps, respectively. The fourth order $R K N_{4}$ integrator is used for the numerical experiments. The results obtained are presented in the left column of Figure 4.2 (which is similar to Figure 3.1a).

Case 2: The Lennard-Jones potential $(r=6, s=12)$. In this case, we integrated until $t=10$ using, approximately, 500( $\varepsilon=0.1), 1000(\varepsilon=0.01), 2000(\varepsilon=0.001)$, and $3000(\varepsilon=0.0001)$ steps, respectively. See the right column of Figure 4.2.

The error formula (4.17) describes certain features of the graphs in Figures 3.1a 


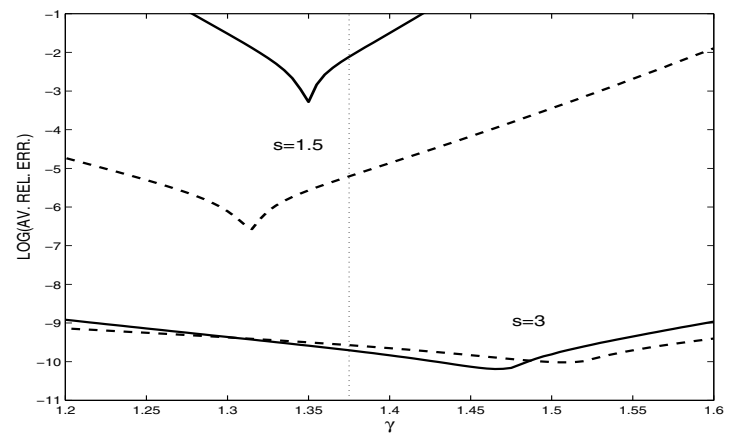

FIG. 4.3. Same as Figure 4.2 for $\varepsilon=0.01$ and 1500 steps (broken lines) and $\varepsilon=0.0001$ and 5000 steps (solid lines) for $s=1.5$ and $s=3$.

and 4.2. In particular, the minimum of the error when $\gamma=\gamma^{*}$ is well approximated. In the two cases considered we have that

$$
\gamma^{*}=\frac{3}{2}-\frac{1}{2 n}<\frac{3}{2} \quad \text { if } r=1, \quad \gamma^{*}=4+\frac{2}{n}>4 \quad \text { if } r=6,
$$

so that in both cases $\gamma^{*}$ approaches the scaling invariant values of $3 / 2$ and 4 , respectively, as $n$ increases. The results also predict that $\log (E)$ should have a linear dependence on $\gamma-\gamma^{*}, n$, and $\log (\varepsilon)$ which is as observed. If $\gamma>\gamma^{*}$, the predicted slope is $\log (E) \sim \log (\varepsilon) \frac{n}{s-r}\left(1+\frac{r}{2}-\gamma\right)$, which is very close to that observed. If $\gamma<\gamma^{*}$, the slope observed is smaller. A possible explanation for this is that the approximation we used for the outer region is valid for $t \in\left[T-\tilde{\varepsilon}^{\frac{1}{2}\left(1+\frac{2}{r}\right)}, T\right]$ with $\tilde{\varepsilon} \geq \varepsilon$. Then $\log (\varepsilon)$ has to be changed by $\log (\tilde{\varepsilon})=\alpha \log (\varepsilon)$ with $\alpha \leq 1$.

Notice also that the performance of the method can drastically change along small intervals of values for $\gamma$ in the monitor function, as observed from the slopes of the curves. In addition, we have found that this slope is proportional to $1 /(s-r)$. To illustrate this dependence with $s$, in Figure 4.3 we repeat the numerical experiments for $r=1$ taking $\varepsilon=0.01$ (broken lines) and $\varepsilon=0.0001$ (solid lines) for $s=1.5$ and $s=3$. The relative slopes are in agreement with the rate $1 /(s-r)$, but their minima have moved from the estimated value $\gamma^{*}=3 / 2-1 / 8$. However, we observed that this value was approximated in the limit $\varepsilon \rightarrow 0$. This is because we have neglected $C(s, r)$ in (4.9), where $C>1$ if $r<s<2 r$ and $C<1$ if $s>2 r$. If we consider $C=$ $\varepsilon^{\log C / \log \varepsilon}$, the minimum obtained will depend on this exponential and its contribution will disappear in the limit $\varepsilon \rightarrow 0$.

4.4. The canonical transformation. The previous error analysis has used backward error analysis and relies on the underlying method being symplectic. It has also implicitly made the assumption that an equal computational cost is incurred for each computational time step. In particular, this arises when we use an explicit method for which the evolution of $T=\frac{1}{2} q^{\gamma} p^{2}$ is exactly computed. When this happens, it must be possible to find a $\mathrm{CT}$ such that $T$ in the new coordinates depends only on the momentum. For the one-dimensional systems considered it is possible to make the transformation. Let us consider the transformation

$$
\left\{\begin{aligned}
Q & =G(q), & & Q^{t}=q^{t} \\
G^{\prime}(q) P & =p, & & P^{t}=p^{t} .
\end{aligned}\right.
$$


Here the function $G(q)$ is defined via the differential equation $G^{\prime}(q)=1 /(a \sqrt{g})$, where $a$ is a suitable constant. The most important feature of this transformation is that it is canonical (from a direct application of Theorem 2.1). Consider now a rescaling function of the form $g(q)=q^{\gamma}$. Then taking $a=2 /(2-\gamma)$ (for simplicity in (4.18)) the CT becomes

$$
\left\{\begin{array} { l } 
{ Q = q ^ { \frac { 2 - \gamma } { 2 } } , } \\
{ P = \frac { 2 } { 2 - \gamma } q ^ { \frac { \gamma } { 2 } } p , }
\end{array} \Leftrightarrow \left\{\begin{array}{l}
q=Q^{\frac{2}{2-\gamma}} \\
p=\frac{2-\gamma}{2} Q^{\frac{\gamma}{\gamma-2}} P .
\end{array}\right.\right.
$$

The Hamiltonian (4.3) in the new coordinates (evolving on the fictive time $\tau$ ) is

$$
K=\frac{(2-\gamma)^{2}}{8} P^{2}+C Q^{\frac{2(\gamma-r)}{2-\gamma}}-Q^{t} Q^{\frac{2 \gamma}{2-\gamma}}
$$

and if we consider $\gamma=1+r / 2$, then

$$
K=\frac{(2-r)^{2}}{32} P^{2}+C Q^{2}-Q^{t} Q^{\frac{4+2 r}{2-r}}
$$

The canonical transformation then has a singularity when $\gamma=r=2$ and this procedure fails, but it can be solved with the CT: $Q=\log (q), P=q p$, with $g=q^{2}$. Observe that the Hamiltonian is now separable in trivial parts, and explicit symplectic RKN integrators can be used.

4.5. Other adaptive methods. The previous examples show the importance of a good choice of scaling function $g$. Now, we study the benefits of using the adaptive and splitting methods described in this paper versus other explicit adaptive geometric (GI) and nongeometric (NGI) integrators, using related scaling functions.

(NGI). First, to show the benefits of using the adaptive symplectic methods versus other standard adaptive nonsymplectic algorithms and symplectic integrators with constant time step, we consider (4.6) with $r=1$ and $s=2$, which corresponds to (1.1). We take as initial conditions $\left(q_{0}, p_{0}\right)=(1,0), \varepsilon=0.1$, and integrate until $t=100$ (40 periods, approximately). The following integrators are considered:

1. The variable time step nonsymplectic ode45 from Matlab with RelTol $=10^{-7}$ and AbsTol $=10^{-10}$ applied to (4.6) (18685 force evaluations).

2. The variable time step variable order nonsymplectic ode113 from Matlab with RelTol $=10^{-10}$ and AbsTol $=10^{-12}$ applied to (4.6) (18361 evaluations).

3. (SI) $R K N_{6}$ applied to (4.6) with $h=\frac{1}{35}$ (38489 evaluations).

4. (ASI) $R K N_{6}$ applied to (4.7) with $\gamma=1.5$ and $\Delta \tau=\frac{1}{6}$ (19019 evaluations). All methods involve approximately the same number of force evaluations except SI, which requires twice this number. The results were shown in Figure 1.1, where the benefits of combining adaptivity and symplecticity are clear.

(GI). Now we compare the efficiency of different adaptive geometric integrators (symmetric and explicit versus symmetric, symplectic, and explicit or implicit). We consider the symmetric second order scheme (2.14) as the basic method and, as in Figure 3.1b, we consider EAV2, EAV4, and EAV6m. We compare the results with those obtained with the new adaptive explicit and splitting methods implemented with $R K N_{4}$ (ARKN4) and $R K N_{6}$ (ARKN6). We consider $r=1, s=2, \varepsilon=0.01$, and $\gamma=1.5$, and the average relative error in energy is measured for different time steps. Figure 4.4 shows the results obtained. The superiority of the symplectic integrators is apparent from these graphs and comes mainly from the fact that they can be 


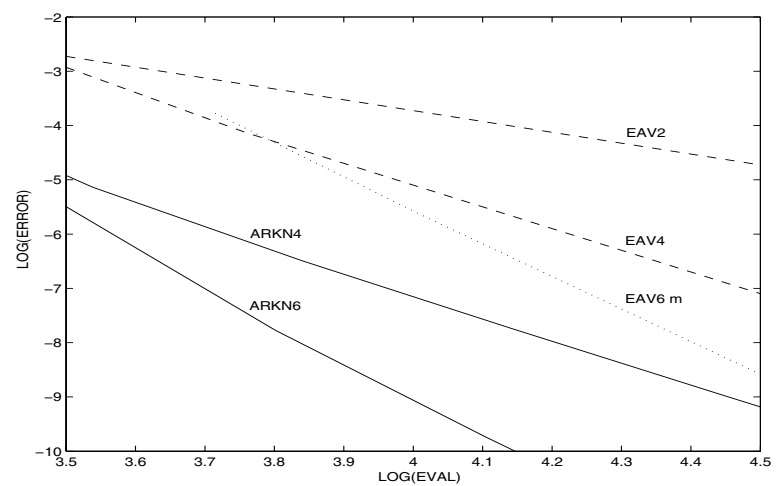

FIG. 4.4. Relative error in energy versus number of evaluations in a $\log -\log$ scale for second, fourth, (dashed lines) and sixth order (dotted line) symmetric methods using (2.14) as the basic method, and fourth and sixth order explicit symplectic RKN methods (solid lines).

implemented with more efficient splitting methods than other methods considered. The computational cost for a fixed number of evaluations is approximately the same for all methods. This is because the ARKN integrators consider the CT, which makes the system very cheap to compute. For problems where this is not possible, the cost of the algorithm has to be considered and then the EAV methods can be superior.

For this one-dimensional problem, (2.15) gives similar results to the nonregularized problem. This is because at the pericenter and apocenter of the orbit, we find that $T(p)=0$ and the time step for the numerical algorithm is the same in those points. This method is not recommended for one-dimensional nonsymmetric problems.

5. Higher dimensional problems. The analysis carried out to find a good regularization function to preserve (or nearly preserve) scaling invariance remains still valid for problems posed in higher dimensions. However, in contrast to the one-dimensional problem, there is not a systematic procedure to solve exactly the Hamiltonian $T=g(\mathbf{q}) \mathbf{p}^{T} \mathbf{p}$ nor to find a CT such that the new Hamiltonian is still easily separable. Nevertheless, it is always possible to use implicit methods to solve this part. For this reason, it is natural to consider important physical problems and to analyze whether it is possible to apply this technique case by case. If this happens, a very efficient method should be obtained. Indeed, the LC regularization is a technique which combines time regularization with a $\mathrm{CT}$, being a standard technique in celestial mechanics for numerically solving the Kepler problem (and perturbations to it) for eccentric orbits. This has been generalized in [2], giving a family of CTs to regularize the equations in two and three dimensions for the potential $V=c / r^{\alpha_{m}}$ with $\alpha_{m}=2(m-1) /(m+1), m=1,2,3, \ldots$ and preserving scale invariance. The case $m=3$ corresponds to the Kepler potential. The goal is to find a CT, $(\mathbf{Q}, \mathbf{P})=\mathcal{C}(\mathbf{q}, \mathbf{p})$ such that

$$
T(\mathbf{q}, \mathbf{p})=g(\mathbf{q}) \mathbf{p}^{T} \mathbf{p}=G(\mathbf{Q}) \mathbf{P}^{T} \mathbf{P}=\bar{T}(\mathbf{Q}, \mathbf{P})
$$

and making $\bar{T}$ easier or cheaper to be numerically computed than $T$. Obviously, the optimal situation corresponds to the case when $G(\mathbf{Q})$ is a constant, but it seems this happens only in few cases. In the following we present the CT of LC/KS for the Kepler potential, and a generalization to another potential. 
5.1. The Levi-Civita/Kustaanheimo-Stiefel transformation. The LC transformation [26] applies to the two-dimensional problem, and its generalization to the three-dimensional space is due to Kustaanheimo-Stiefel (KS) [24, 38]. It corresponds to the Hamiltonian (2.10) with the regularization function $g(\mathbf{q})=r$, with $r=\left(\mathbf{q}^{T} \mathbf{q}\right)^{1 / 2}$. This transformation is especially suitable for the numerical analysis of highly eccentric orbits in the Kepler problem (for which $V=-1 / r$ ) as the singularity in the potential is removed. As in the one-dimensional problem, the original Hamiltonian $H$ is separable, but the transformed Hamiltonian $K$ contains the term $\frac{1}{2} r \mathbf{p}^{T} \mathbf{p}$, which is exactly solvable. However, it is computationally expensive, and the benefits of using splitting methods break down. However, there is a simple solution. Kustaanheimo and Stiefel [24, 38] proposed the CT

$$
\mathbf{q}=\hat{Q} \mathbf{Q}, \quad \mathbf{p}=\frac{1}{2 R^{2}} \hat{Q} \mathbf{P} .
$$

Here, the three-dimensional system has been enlarged with another dimension, using the vectors $\mathbf{q}=\left(q_{1}, q_{2}, q_{3}, 0\right)^{T}$ and $\mathbf{p}=\left(p_{1}, p_{2}, p_{3}, 0\right)^{T}$. Now $\mathbf{Q}=\left(Q_{1}, Q_{2}, Q_{3}, Q_{4}\right)^{T}$, $\mathbf{P}=\left(P_{1}, P_{2}, P_{3}, P_{4}\right)^{T}$ are the KS-variables, $R=\left(\mathbf{Q}^{T} \mathbf{Q}\right)^{1 / 2}$, and $\hat{Q}$ is the KS-matrix

$$
\hat{Q}=\left(\begin{array}{cccc}
Q_{1} & -Q_{2} & -Q_{3} & Q_{4} \\
Q_{2} & Q_{1} & -Q_{4} & -Q_{3} \\
Q_{3} & Q_{4} & Q_{1} & Q_{2} \\
Q_{4} & -Q_{3} & Q_{2} & -Q_{1}
\end{array}\right)
$$

This includes the LC transformation, which corresponds to the particular case $q_{3}=$ $p_{3}=Q_{3}=Q_{4}=P_{3}=P_{4}=0$.

If we substitute this transformation into the Hamiltonian (2.10) with $V=-1 / r$ and consider that $\mathbf{Q}^{T} \mathbf{Q}=R^{2}$ and $\hat{Q}^{T} \hat{Q}=R^{2} I$ with $I$ is the identity matrix, we get the following Hamiltonian $K$ in the new coordinates:

$$
K=\frac{1}{8} \mathbf{P}^{T} \mathbf{P}-1-R^{2} Q^{t}
$$

Since $Q^{t}=H_{0}$ is constant, this is a harmonic oscillator which is easy and cheap to integrate even for highly eccentric orbits. One problem for this regularization is that it does not preserve the scaling invariance of the Kepler problem. From the one-dimensional problem we know the influence of the regularization function on the performance of a numerical integrator as well as its close relation with the scaling invariance. Then, as we will show in one example, the performance of the LC/KS regularization deteriorates for some problems. For this reason, the LC/KS transformation is generalized in [2] in order to allow the use of a regularization function which both removes the singularity of the Kepler problem and still preserves its scaling invariance.

5.2. The Lennard-Jones potential. Let us consider the Hamiltonian in the two- and three-dimensional space

$$
H=\frac{1}{2} \mathbf{p}^{T} \mathbf{p}-\frac{a}{r^{6}}+\frac{b}{r^{s}},
$$

with $s>6, a=c \beta^{6}, b=c \beta^{s}$, and $c, \beta$ constants. The term $-a / r^{6}$ gives usually the dominant contribution for most evolution region, and a regularization function which makes this part scaling invariant is, as before, $g=r^{4}$. Unlike in the previous 
case, we present a CT which works for this important potential and function $g$. The Hamiltonian (5.3) with $s=12$ is frequently used in molecular dynamics to describe the two-body interaction in the $N$-body problem. But, the CT we propose works only for the two-body problem, and has to be considered as an illustrative example in the search for Hamiltonians $T=g(\mathbf{q}) \mathbf{p}^{T} \mathbf{p}$ with exact solution. Its possible application to the $N$-body problem must be considered as an open problem.

Let us now consider the coordinate transformation $\mathbf{q}=\Phi(\mathbf{Q})$ with

$$
\Phi(\mathbf{Q})=\frac{1}{R^{2 m}} \mathbf{Q}
$$

Then

$$
\Phi^{\prime}(\mathbf{Q})_{i, j}=\frac{1}{R^{2(m+1)}}\left(R^{2} \delta_{i j}-2 m Q_{i} Q_{j}\right) .
$$

A simple inspection of (5.5) indicates that $\Phi^{\prime}$ is orthogonal only for $m=1$, and this is the only case we consider, where

$$
\Phi^{\prime} \Phi^{\prime T}=\frac{1}{R^{4}} I
$$

Let us denote $\hat{Q}_{i j}=\left(R^{2} \delta_{i j}-2 Q_{i} Q_{j}\right), \hat{q}_{i j}=\left(r^{2} \delta_{i j}-2 q_{i} q_{j}\right)$, where $r=1 / R, \hat{Q}^{T} \hat{Q}=$ $R^{4} I, \hat{q}^{T} \hat{q}=r^{4} I$. Then, from Theorem 2.1 and (5.4)-(5.6) with $m=1$, it is immediate that the transformation

$$
\left\{\begin{array} { l } 
{ \mathbf { q } = \frac { 1 } { R ^ { 2 } } \mathbf { Q } , } \\
{ \mathbf { p } = \hat { Q } \mathbf { P } , }
\end{array} \Leftrightarrow \left\{\begin{array}{l}
\mathbf{Q}=\frac{1}{r^{2}} \mathbf{q}, \\
\mathbf{P}=\hat{q} \mathbf{p}
\end{array}\right.\right.
$$

is canonical, for both the two- and three-dimensional problem (it is not necessary to introduce a new coordinate for the three-dimensional problem, as in the KS transformation). In addition, since $r^{4} \mathbf{p}^{T} \mathbf{p}=\mathbf{P}^{T} \mathbf{P}$, this CT makes separable the LennardJones Hamiltonian with $g=r^{4}$ (which makes the system nearly scaling invariant), and the Hamiltonian to solve is

$$
K_{s c}=\frac{1}{2} \mathbf{P}^{T} \mathbf{P}-a R^{2}+b R^{s-4}+\frac{Q^{t}}{R^{4}} .
$$

If the $\mathrm{LC} / \mathrm{KS}$ regularization is considered, the resulting Hamiltonian is

$$
K_{L C}=\frac{1}{8} \mathbf{P}^{T} \mathbf{P}-\frac{a}{R^{10}}+\frac{b}{R^{2 s-2}}+Q^{t} R^{2},
$$

which contains singularities of higher order.

\section{Numerical examples of two-dimensional problems.}

6.1. The two-dimensional Lennard-Jones problem. We consider (5.3) with $s=12$ and initial conditions $q_{1}=1 / 5, q_{2}=0, p_{1}=0, p_{2}=\sqrt{2\left(H_{0}-V(r)\right)}$, for $H_{0}-V(r) \geq 0$, which corresponds to a system with energy $H_{0}$. We take $c=20$, $\beta=0.15,0.1$, and $H_{0}=-0.1$, and we integrate until $t=5$. We study the performance of the schemes taking $g=r^{m}$ in the following cases: (i) nonregularization $(m=0)$; (ii) LC regularization $(m=1)$; and (iii) scaling invariant regularization $(m=4)$. We measured the average relative error in energy versus the number of force evaluations for 

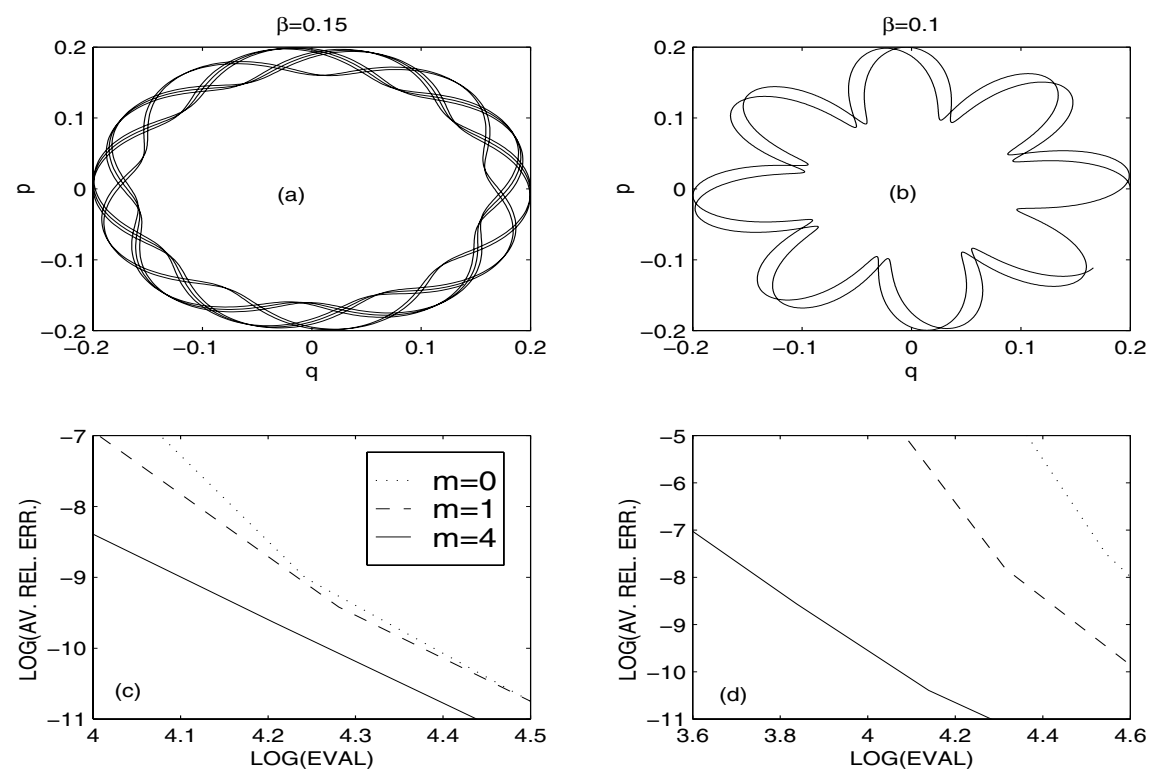

FIG. 6.1. (a), (b): trajectories for the Lennard-Jones problem. (c), (d): average relative error in energy versus number of evaluations in a $\log -\log$ scale when nonregularization is considered $(m=0)$, when considering the $L C$ regularization $(m=1)$, and when considering the scaling invariant regularization $(m=4)$.

different time steps $\Delta \tau$. In all cases, we integrated the systems using the symplectic $R K N_{6}$ integrator. Figure 6.1 shows the trajectory of the orbits and the average relative error in energy of the methods versus the number of evaluations. We observe that for $\beta=0.1$ the trajectory approaches closer to the origin and the scaling invariant scheme with $g=r^{4}$ is much superior. We clearly observe that the case $m=1$ does not play any special role as it does in the Kepler problem, giving less efficient results. Then, for this problem, the choice $m=4$ gives the most efficient algorithm, and the relative performance will increase in the limit $\beta \rightarrow 0$. This superiority is also observed numerically for other values of $s$ and initial conditions (consistently with the results obtained for the one-dimensional problem).

6.2. A perturbed Kepler problem. Let us now consider the two-dimensional perturbed Kepler problem

$$
H=\frac{1}{2}\left(p_{1}^{2}+p_{2}^{2}\right)-\frac{1}{r}+\frac{\varepsilon}{r^{3}},
$$

which, among others, describes in first approximation the dynamics of a satellite moving into the gravitational field produced by a slightly oblate planet. This problem is neither integrable nor scaling invariant, although it does have an approximate scaling symmetry if $\varepsilon / r^{3}$ is small. We take as initial conditions $q_{1}=1-e, q_{2}=0, p_{1}=$ $0, p_{2}=\sqrt{(1+e) /(1-e)}$ which, for the unperturbed problem, would correspond to an orbit of period $2 \pi$, eccentricity $e$, and energy $-1 / 2$. We integrate until $t=10 \pi$ for different values of $e$ using the $R K N_{4}$ integrator, and measure the average relative error in energy choosing the fictive time steps such that the whole integration is carried out with approximately 100 steps. We consider two cases: (i) the nonregularized 


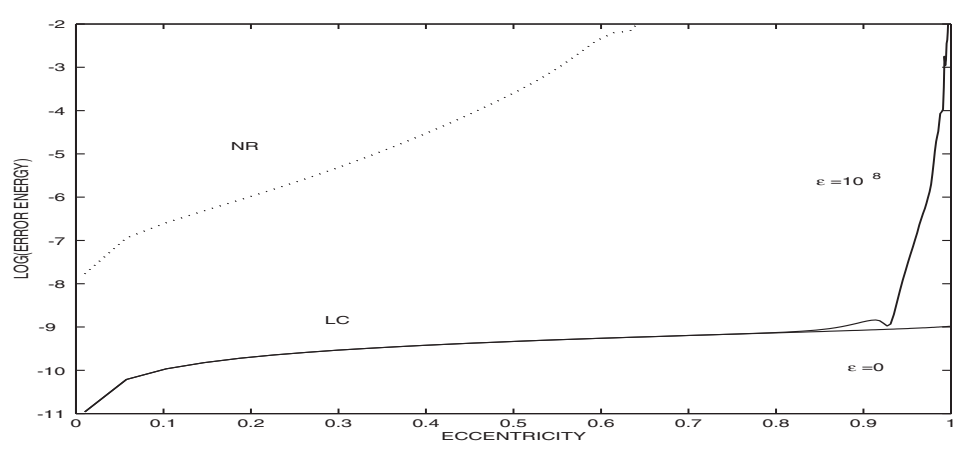

FIG. 6.2. Average relative error in energy in logarithmic scale as a function of the eccentricity for the nonregularized system and for the $L C$ regularization using the same fictive time step. This is done for $\varepsilon=0$ and $\varepsilon=10^{-8}$.

case (NR) and (ii) the LC regularization. We started the experiments with $\varepsilon=0$ (unperturbed problem) and repeated the experiment with $\varepsilon=10^{-8}$. The results are shown in Figure 6.2 where three important facts can be deduced: (i) when using LC regularization the error does not deteriorate at high eccentricities if $\varepsilon=0$; (ii) the performance of the regularized scheme is considerably superior to the constant step size integrator, even for small eccentricities; (iii) even for a very small perturbation the performance of LC is severely deteriorated for high eccentricities.

The reason for the good performance of the LC regularization on the unperturbed Kepler $(\varepsilon=0)$ problem comes because the Hamiltonian to be solved is (5.2), which is exactly solvable. This is possible because the original system is also integrable. The symplectic integrators used solve exactly a perturbed harmonic oscillator or, equivalently, they solve exactly a Kepler problem, with slightly different parameters, giving also a closed trajectory (this is a surprising fact since it is well known that most symplectic integrators, when applied to the Kepler problem, give trajectories with a precession of the orbit). However, if some perturbations are introduced making the system not integrable, the performance of the $\mathrm{LC} / \mathrm{KS}$ regularization can be seriously deteriorated for high eccentricities. The main reason is because the fictive time is not (nearly) scaling invariant. In addition, we must remember that this excellent performance for the LC/KS regularization happens only when solving the Kepler problem in Cartesian coordinates, but not in polar coordinates as shown in the onedimensional example of section 4 .

7. Conclusions. In this paper we present a procedure to build adaptive geometric integrators making use of the Poincaré transformation. For the one-dimensional problem, a backward error analysis for a family of problems is presented, using adaptive splitting methods, and an explicit dependence of the error with the monitor function, $g$ (at constant work), is obtained. The error and the optimal function, $g$, strongly depend on the order of the method used and, most importantly, on the value which makes the regularized system scaling invariant (for the dominant part of the potential). These results perfectly agree with those obtained from the numerical experiments and can be extrapolated to other higher dimensional problems. Then the choice of the correctly scaled monitor function is essential for the performance of the method. In those cases where the Hamiltonian $T=g(\mathbf{q}) \mathbf{p}^{T} \mathbf{p}$ is exactly solvable using a proper $\mathrm{CT}$, explicit splitting methods can be used, which can be considered 
a generalization of the $\mathrm{LC} / \mathrm{KS}$ regularization. As an example, an adaptive explicit and symplectic method for the two- and three-dimensional Lennard--Jones potential is presented, showing its superiority versus other regularizations. We also show how the well-known LC/KS regularization (which does not preserve scaling invariance for the Kepler problem) looses its efficiency on some problems.

Obviously, it is not always possible to find a CT for each function $g$. In such a case, several alternatives can be considered. For example: (i) to consider the function $g$ and its corresponding CT which are closer to the optimal scaling function obtained from the error analysis; (ii) choose this optimal function and either use an implicit method to solve the transformed kinetic energy or use the explicit adaptive Verlet method (2.14) (or consider it the basic method to higher order composition integrators). In the last case, the previous backward error analysis does not apply but, from Figure 3.1b, we expect the results obtained are still approximately valid.

\section{REFERENCES}

[1] G.I. Barenblatt, Scaling, Self-similarity and Intermediate Asymptotics, Cambridge University Press, Cambridge, UK, 1996.

[2] S. Blanes AND C.J. BudD, Explicit Adaptive SYmplectic (EASY) integrators: A scaling invariant generalisation of the Levi-Civita and $K S$ regularisations, Celestial Mech. Dynam. Astronom. 89 (2004), pp. 383-405.

[3] S. Blanes, F. Casas, And J. Ros, Symplectic integration with processing: A general study, SIAM J. Sci. Comput., 21 (1999), pp. 711-727.

[4] S. Blanes, F. Casas, And J. Ros, Processing symplectic methods for near-integrable Hamiltonian systems, Celestial Mech. Dynam. Astronom., 77 (2000), pp. 17-35.

[5] S. Blanes, F. CAsas, AND J. Ros, High-order Runge-Kutta-Nyström geometric methods with processing, Appl. Numer. Math., 39 (2001), pp. 245-259.

[6] S. Blanes And P.C. Moan, Practical symplectic partitioned Runge-Kutta and Runge-KuttaNyström methods, J. Comput. Appl. Math., 142 (2002), pp. 313-330.

[7] S.D. Bond AND B. LEIMKUHLER, Time-transformations for reversible variable stepsize integration, Numer. Algorithms, 19 (1998), pp. 55-71.

[8] C.J. Budd, B. Leimkuhler, And M.D. Piggott, Scaling invariance and adaptivity, Appl. Numer. Math., 39 (2001), pp. 261-288.

[9] C.J. BudD AND M.D. PIGGOTT, The geometric integration of scale-invariant ordinary and partial differential equations, J. Comput. Appl. Math., 128 (2001), pp. 399-422.

[10] C.J. Budd AND M.D. PIGGotT, Geometric integration and its applications, Handb. Numer. Anal., North-Holland, Amsterdam, 2003.

[11] C.J. Budd, W. Huang, And R.D. Russell, Moving mesh methods for problems with blow-up, SIAM J. Sci. Comput., 17 (1996), pp. 305-327.

[12] M.P. CALvo, High order starting iterates for implicit Runge-Kutta methods: An improvement for variable-step symplectic integrators, IMA J. Numer. Anal., 22 (2002), pp. 153-166.

[13] M.P. Calvo, J.M. Sanz-Serna, And M.A. López-Marcos, Variable step implementations of geometric integrators, Appl. Numer. Math., 28 (1998), pp. 1-16.

[14] M.P. Calvo And J.M. SAnZ-SERna, The development of variable-step symplectic integrators, with application to the two-body problem, SIAM J. Sci. Comput., 14 (1993), pp. 936-952.

[15] S. Cirilli, E. Hairer, And B. Leimkuhler, Asymptotic error analysis of the adaptive Verlet method, BIT, 39 (1999), pp. 25-33.

[16] M. Creutz And A. Gocksch, Higher-order hybrid Monte Carlo algorithms, Phys. Rev. Lett., 63 (1989), pp. 9-12.

[17] B. Gladman, M. Duncan, And J. Candy, Symplectic integrators for long-term integrations in celestial mechanics, Celestial Mech. Dynam. Astronom., 52 (1991), pp. 221-240.

[18] H. Goldstein, Classical Mechanics, 2nd ed., Addison-Wesley, Reading, MA, 1980.

[19] E. HAIRER, Variable time step integration with symplectic methods, Appl. Numer. Math., 25 (1997), pp. 219-227.

[20] E. Hairer, Ch. Lubich, AND G. WAnner, Geometric Numerical Integration: StructurePreserving Algorithms for Ordinary Differential Equations, Springer Series in Computational Mathematics 31, Springer-Verlag, Berlin, 2002.

[21] T. Holder, B. Leimkuhler, And S. Reich, Explicit variable step-size and time-reversible 
integration, Appl. Numer. Math., 39 (2001), pp. 367-377.

[22] W. Huang and B. Leimkuhler, The adaptive Verlet method, SIAM J. Sci. Comput., 18 (1997), pp. 239-256.

[23] A. Iserles, A First Course in the Numerical Analysis of Differential Equations, Cambridge University Press, Cambridge, 1996.

[24] P. KustaAnheimo And E. Stiefel, Perturbation theory of Kepler motion based on spinor regularization, J. Reine Angew. Math., 218 (1965), pp. 204-219.

[25] B. LeIMKUHLeR, Reversible adaptive regularization: Perturbed Kepler motion and classical atomic trajectories, R. Soc. Lond. Philos. Trans. Ser. A Math. Phys. Eng. Sci., 357 (1999), pp. 1101-1133.

[26] T. Levi-Civita, Sur la regularization du problème des trois corps, Acta Math., 42 (1920), pp. 99-144.

[27] J.E. Marsden, Lectures on Mechanics, Cambridge University Press, Cambridge, UK, 1992.

[28] R.I. MCLACHLAN, On the numerical integration of ordinary differential equations by symmetric composition methods, SIAM J. Sci. Comput., 16 (1995), pp. 151-168.

[29] R.I. MCLAChlan, Composition methods in the presence of small parameters, BIT, 35 (1995), pp. $258-268$.

[30] R.I. McLachlan and R. Quispel, Splitting methods, Acta Numer., 11 (2002), pp. 341-434.

[31] S. Mikkola, Practical symplectic methods with time transformation for the few-body problem, Celestial Mech. Dynam. Astronom., 67 (1997), pp. 145-165.

[32] S. Mikkola And K. TANiKawa, Explicit symplectic algorithms for time-transformed Hamiltonians, Celestial Mech. Dynam. Astronom., 74 (1999), pp. 287-295.

[33] M.D. Piggott, Geometric integration of differential equations, Ph.D. Thesis, University of Bath, Bath, UK, 2002

[34] M. Preto and S. Tremaine, A class of symplectic integrators with adaptive time step for separable Hamiltonian systems, Astron. J., 118 (1999), pp. 2532-2541.

[35] S. REICH, Backward error analysis for numerical integrators, SIAM J. Numer. Anal., 36 (1999), pp. 1549-1570.

[36] J.M. Sanz-Serna And M.P. Calvo, Numerical Hamiltonian Problems, Chapman and Hall, London, 1994.

[37] R. Skeel, Variable step size destabilizes the Störmer/leapfrog/Verlet method, BIT, 33 (1993), pp. $172-175$.

[38] E.L. Stiefel ANd G. Scheifel, Linear and Regular Celestial Mechanics, Springer-Verlag, Berlin, 1971.

39] D. Stoffer, Variable steps for reversible integration methods, Computing, 55 (1995), pp. 1-22.

[40] M. Suzuki, Fractal decomposition of exponential operators with applications to many-body theories and Monte Carlo simulations, Phys. Lett. A, 146 (1990), pp. 319-323.

[41] C. Sweet and B. Leimkuhler, Private communication.

[42] J. Waldvogel, A new regularization of the planar problem of three bodies, Celestial Mech., 6 (1972), pp. 221-231.

[43] J. Wisdom And M. Holman, Symplectic maps for the N-body problem, Astron. J., 102 (1991), pp. 1528-1538.

[44] H. YoshidA, Construction of higher order symplectic integrators, Phys. Lett. A, 150 (1990), pp. 262-268. 